\title{
䬤pubvet
}

https://doi.org/10.31533/pubvet.v13n10a429.1-7

\section{Medicina veterinária de desastres e catástrofes: plano de ação}

\author{
Marcos Vinícius de Souza ${ }^{1 *} \bullet$ \\ ${ }^{I}$ Prof. Dr. Docente do Curso de Graduação em Medicina Veterinária e Medicina no Centro Universitário UNIFACIG. Rua Darcy César de \\ Oliveira Leite, 600, Alfa Sul, Manhuaçu, MG, 36904-219, Brasil. \\ *Autor para correspondência, E-mail: mvscardoso@yahoo.com.br
}

Resumo. Com a intervenção cada vez maior do homem sobre os ecossistemas, muitos são os riscos criados para a vida humana e dos animais. Assim, diversas situações exigem atitudes e responsabilidades por parte da sociedade, a partir do momento que o poder sobre a natureza se torna mais evidente. Fica clara a necessidade de se especializar o conhecimento acerca de tais situações, o que tem forçado o desenvolvimento da especialidade de "medicina veterinária de catástrofe", uma vez que esse ramo de atuação é bem distinto de outras formas de atividades veterinárias. A medicina veterinária em catástrofes pode atuar de forma isolada ou ser paralela e complementar a outras, realizadas por organizações médicas de outros ramos, civis, públicas, ou não governamentais, devendo ser capaz de se articular com qualquer uma delas.

Palavras-chave: Busca e salvamento, resgate animal, emergência, intervenção, gestão

\section{Veterinary medicine in disaster and catastrophe: action plan}

Abstract. With man interfering more and more on ecosystems, many are the risks generated for human and animal life. Therefore, there are many situations that demand attitudes and responsibilities by society, from the moment that the power over nature becomes more evident. It is clear the necessity of specializing knowledge about these situations, which has forced the development of the specialty "veterinary medicine of catastrophe", as this action field is very different from the other veterinarian activities. The veterinary medicine of catastrophe can act in isolation or be parallel and complementary to others, carried out by medical organizations from other branches, civil, public, or nongovernmental, and being able to articulate with any of them.

Keywords: Search and rescue, animal rescue, emergency, intervention, management

\section{Medicina veterinaria de desastres y catástrofes: plano de acción}

Resumen. Con el aumento de la intervención humana en los ecosistemas, se crean muchos riesgos para la vida humana y animal. Por lo tanto, diversas situaciones requieren actitudes y responsabilidades de la sociedad, a medida que el poder sobre la naturaleza se hace más evidente. Queda clara la necesidad de especializar el conocimiento sobre tales situaciones, lo que ha forzado el desarrollo de la especialidad de "medicina veterinaria catastrófica", ya que esta rama de la práctica es muy distinta de otras formas de actividades veterinarias. La medicina veterinaria en casos de desastre puede actuar aisladamente o ser paralela y complementaria a otras, realizada por organizaciones médicas de otras ramas, civiles, públicas o no gubernamentales, y debe poder articularse con cualquiera de ellas.

Palabra clave: Rescate de animales, búsqueda de emergencia, emergencia, intervención, gestión 


\section{Introdução}

No ano de 2013 ocorreram 330 desastres naturais, quantidade mais baixa da última década. O total de humanos afetados foi de 96,5 milhões, também inferiores quando comparado com a média anual de 215,8 milhões no último decénio (Guha-Sapir et al., 2013). Esses dados não incluem animais e por esse motivo deve ser questionado se esses seres "têm lugar na arca". Em caso afirmativo, deve vingar o imperativo moral de salvá-los protegendo igualmente os seus tutores do peso emocional associado à perda do(s) seu(s) companheiro(s); porém, são motivos econômicos, éticos, emocionais e ambientais que ditarão a resposta (Irvine, 2009). A Organização Mundial de Saúde (OMS) define como catástrofe qualquer acontecimento que cause estragos, desestabilização econômica, perda de vidas humanas e deterioração de saúde, a uma escala tal, que justifique uma mobilização excepcional de auxílios vindos de fora da comunidade e da zona atingida e enfatiza o elemento específico da Medicina de Catástrofe: a insuficiência temporária de recursos para responder às necessidades (Bandeira, 2008).

Segundo a World Association for Disaster and Emergency Medicine (WADEM) a Medicina de Catástrofes tem como objetivos (Sundnes \& Birnbaum, 2003):

- Reverter os efeitos negativos sobre a saúde provocados pelo incidente;

- Modificar o perigo responsável pelo incidente;

- Reduzir a vulnerabilidade da sociedade para eventos futuros e

- Melhorar a preparação para a resposta a incidentes futuros, tendo como referência a catástrofe em um processo com várias fases.

Sendo as situações de catástrofes tão diversas nas suas variáveis, têm sido propostas algumas classificações. Umas visam sistematizar os riscos, outras, mais complexas, apresentam-se numa perspectiva de planejamento de resposta às consequências, tendo em conta a sua duração (Curtas: < 1 hora, Média: entre 6 e 24 horas e Longas: mais de 24 horas), extensão (Raio de até $1 \mathrm{~km}$, Raio entre 1 e 100 km e Raio > 100 km) e o número de vítimas (Menor: entre 25 e 100 casos, Moderada: entre 100 e 1000 casos e Maior > 1000 casos (Bandeira, 2008). Nessa perspectiva, a catástrofe não é um fenômeno absoluto, mas uma relação entre as necessidades e os recursos disponíveis, sendo essa a relação que faz a diferença entre um incidente limitado e uma grande catástrofe.

Os organismos internacionais, como a OMS e o Pan-American Health Organization (PAHO, 1981), propõem uma divisão por fase apenas após a ocorrência e que servem de base à atual tipologia do socorro, tendo em conta o tempo e as necessidades previsíveis, podendo ser resumidos em:

Fase 1 - Emergência: Devem ser providenciados cuidados iniciais de emergência médica, incluindo Técnicas de Suporte de Vida em Situações de Trauma (Advance Trauma Life Support - ATLS ${ }^{\odot}$ ), terá de ser autossustentável, estar no terreno até 24 horas após o incidente, sendo necessária à sua ação durante 48 horas.

Fase 2 - Acompanhamento Médico e Trauma: Deve ser providenciado acompanhamento médico e de trauma, durante a fase em que o sistema de saúde não tem capacidade de resposta às solicitações. Deve estar operando entre o terceiro e o quinto dia, com um conjunto específico de especialidades e ser capaz de se manter durante um período máximo de 15 dias, com um suporte mínimo das estruturas locais.

Fase 3 - Infraestrutura de saúde temporária: Deve ser providenciado o apoio médico e cirúrgico durante a fase de reconstrução.

O apoio veterinário aos animais afetados partirá sempre de uma avaliação que detecte as necessidades locais, e para que seja o mais adequado terá necessariamente que haver uma adaptação logística e das equipes envolvidas. Tais equipes serão responsáveis por organizar e apoiar o resgate e evacuação animal, instalar pontos de triagem que permitam precisar quais os feridos e doentes que poderão ser tratados localmente, quais têm de ser transportados para centros de atendimento médico veterinário com mais estrutura e capacidade de resposta, e quais podem ser direcionados de imediato para alojamentos temporários previamente construídos. Relativamente a esses alojamentos, será necessário assegurar o correto manejo dos animais neles alojados e, periodicamente, sujeitá-los a inspeções que visem à garantia da saúde, segurança e bem-estar animal (United States Department of Health \& Human Services, 2015). 
A intervenção em situações de emergência e desastre, segundo Ventura (2011) é composta por três etapas: preventiva, emergencial e pós-emergencial. Pode soar estranho falar em prevenção quando se trata de eventos inesperados ou mesmo imprevisíveis. Contudo, em diversos aspectos, pode-se reduzir a probabilidade de ocorrência de desastres e/ou amenizar as consequências que o mesmo terá. Incluemse aí ações dirigidas a avaliar e reduzir riscos e uma preparação coletiva, como costuma acontecer nas regiões comumente atingidas por terremotos, por exemplo. Estamos falando, portanto, de uma questão de políticas públicas, mas para além dos objetivos já citados, políticas de Estado que proporcionem condições mínimas para uma vida digna, compondo uma rede efetiva de serviços e recursos acessíveis.

O objetivo deste trabalho é propor um plano de ação para subsidiar a Medicina Veterinária para Casos de Desastres e Catástrofes, de forma a aperfeiçoar a sua capacidade de resposta perante uma situação de calamidade, em território nacional.

\section{Plano de ação}

Pode-se constatar que o Brasil dispõe de um conjunto de recursos humanos e materiais bastante significativos e sua insuficiência num determinado momento e situação estão mais relacionados com a dificuldade de planejamento do que com a sua escassez, propriamente dita.

Para se conseguir uma resposta eficaz são necessários os seguintes elementos:

\section{1 - Equipe de reconhecimento e segurança médica veterinária (ERSMV)}

As ERSMV são constituídas por profissionais habilitados que realizam o reconhecimento, quer do ponto de vista das infraestruturas danificadas ou em risco, quer da previsão do número de animais vitimados, da sua localização e das condições para o seu socorro, devendo para tal ser utilizado um inventário sistematizado, como por exemplo, a classificação do incidente, localização exata, a hora do incidente, tipos de perigos presentes e potenciais, tipos e vias de acesso, número de animais vitimados e capacidades de apoio existentes.

\section{2 - Equipe de busca e salvamento médico veterinário "Search and Rescue" (EMV SAR)}

Essas equipes são igualmente constituídas por profissionais habilitados, com capacidades técnicas que lhe permitem atender os animais vitimados em locais com infraestrutura precária. São integradas por médicos veterinários com formação em resgate e, caso a situação no terreno permita, poderá também existir auxiliares nessa equipe.

As funções das EMV SAR consistem na triagem primária dos animais vitimados, o seu transporte em condições mínimas de estabilidade para o Ponto de Recolhimento de Animais Feridos (PRAF). No PRAF deverá haver um médico veterinário de prontidão para realizar o encaminhamento dos animais feridos, a partir da triagem efetuada, para a área de evacuação, ou para o Centro de Recolhimento Avançado de Saúde Animal (CRASA), onde ficarão sob responsabilidade da Equipe Móvel de Saúde Animal (EMSA), devendo ser iniciado o preenchimento da Ficha Clínica de Emergência Animal (FCEA).

\section{3- Equipes móveis de saúde animal (EMSA)}

Essas equipes são constituídas por dois médicos veterinários e as suas funções consistem no apoio dos animais vitimados na área do PRAF, incluindo o suporte básico de vida e imobilização primária, bem como no seu transporte em condições de estabilidade, de acordo com o princípio da continuidade de cuidados, para o Centro de Recolhimento Avançado de Saúde Animal (CRASA).

\section{4 - Centro de recolhimento avançado de saúde animal (CRASA)}

Essa estrutura corresponde a um centro médico veterinário, sendo constituída basicamente por nove áreas funcionais:

- $\quad$ Triagem de Campo Médica Veterinária: constituído por uma ou mais unidades móveis e equipamentos adequados, devendo estar sob supervisão de um médico veterinário com experiências em trabalhos de campo (por exemplo, levantamento e resgate de fauna) e um com formação em emergência. 
A função dessa(s) equipe(s) é categorizar os animais (Tabela 1) que serão enviados ao CRASA para que não haja um esgotamento de recursos ou lotação das instalações.

Durante a triagem serão encontrados animais contaminados com agentes biológicos e /ou químicos, de forma que se deve iniciar o processo de descontaminação. O processo visa a remoção e/ou inativação dos agentes contaminantes de forma a diminuir a absorção e prevenir o surgimento de alterações sistêmicas, além de evitar a contaminação secundária à equipe envolvida e a outros animais resgatados.

Tabela 1. Triagem de campo médica veterinária, de acordo com o estado do paciente.

\begin{tabular}{ccl}
\hline Código & Categoria & Descrição \\
\hline & Morto, agonizante ou eutanásia* & Ferimentos mortais ou não compatíveis com a "qualidade de vida". Eutanásia* \\
& Imediato & Problemas no sistema respiratório, cardiovascular, termorregulatório \\
& Urgente & Problemas no sistema cardiovascular, musculoesquelético, neurológico, feridas abdominais \\
& Menor & Problemas no sistema musculoesquelético, neurológico, feridas abdominais \\
\hline
\end{tabular}

Fonte: Adaptado de Vieira (2016). *Conforme Resolução nº 1000 do CFMV de 11 de maio de 2012.

- $\quad$ Triagem Médica Veterinária: constituída por uma unidade e equipamentos logísticos de apoio, devendo estar sob supervisão de um médico veterinário com formação em emergência, bem como um intensivista. A função desse setor consiste em identificar e classificar os animais admitidos individualmente; realizar uma triagem secundária (frequência respiratória, frequência cardíaca, pressão arterial, temperatura, score corporal, nível de hidratação/desidratação, condição neurológica e ferimentos); completar ou inserir os dados na FCEA; iniciar ou dar continuidade aos cuidados médicos veterinários e destinar aos setores e/ou unidades adequados (Tabela 2).

Tabela 2. Triagem Médica Veterinária.

\begin{tabular}{ccl}
\hline Código & Categoria & \multicolumn{1}{c}{ Descrição } \\
\hline & Morto, agonizante ou eutanásia* & Ferimentos mortais ou condição neurológica intensamente alterada \\
Imediato & Frequência respiratória, frequência cardíaca, pressão arterial e temperatura anormais \\
& Urgente & Frequência cardíaca, pressão arterial e condição neurológica anormais \\
& Menor & $\begin{array}{l}\text { Ferimentos menores, frequência respiratória, frequência cardíaca, pressão arterial, } \\
\text { temperatura, hidratação, score corporal e condição neurológica normais }\end{array}$ \\
\hline
\end{tabular}

Fonte: Adaptado de Vieira (2016). *Conforme Resolução nº 1000 do CFMV de 11 de maio de 2012.

Animais classificados com o código preto, para além dos mortos ou agonizantes, são os que não apresentam melhora clínica ou que tenham lesões que exigem cuidados para os quais os recursos são inexistentes. Nesses casos a eutanásia deve ser ponderada e, quando for a opção, ser documentada. Pacientes com o código vermelho e amarelo são tratados no momento e aos que for atribuído o código verde, serão observados periodicamente em uma área destinada a esse fim (Ryan, 2008; Wingfield, 2009; Wingfield \& Palmer, 2009).

- $\quad$ Unidades de Descontaminação Médica Veterinária (EDMV): Constituídas por uma unidade para grandes animais e uma unidade para pequenos animais, além de equipamentos adequados, devendo estar sob supervisão de um médico veterinário epidemiologista e um voltado à medicina veterinária do coletivo. Essa unidade visa criar diretrizes e procedimentos para que a descontaminação seja bem-sucedida.

As unidades de descontaminação devem ser montadas em zonas conforme sugerido por Aires et al. (2015). A delimitação das zonas é feita tendo em conta a carga de contaminantes existente: a zona quente é a zona contaminada, onde só se admite a presença de pessoal devidamente equipado, e de onde não podem sair pessoas e animais sem serem descontaminados. A zona morna é o local que liga as zonas quente e fria, na qual será montado o corredor de redução de descontaminação que, por sua vez, é o local onde se realiza a descontaminação propriamente dita. Tal como na zona quente, só se admite a presença de pessoal com EPI adequado. A zona mais exterior é a zona fria, externa ao acidente e onde o risco é mínimo ou ausente. É nessas que se dá a maior parte das atividades relacionadas com o evento e onde estão as equipes de apoio, incluindo a assistência médico veterinária e o alojamento dos animais já descontaminados. Embora o risco de exposição seja muito reduzido nessa zona, só o pessoal indispensável deve estar presente (Aires et al., 2015; Wingfield, 2009; Wingfield \& Palmer, 2009). 
- $\quad$ Unidade de Terapia Intensiva Animal (UTIA) e Cirurgias de Baixa Complexidade: Constituída por uma unidade e equipamentos adequados à função, devendo estar sob a supervisão de um médico veterinário intensivista e um cirurgião geral. Essa unidade deve garantir procedimentos de reanimação, estabilização e monitorização; além de realizar pequenas cirurgias e tratamentos ortopédicos conservativos.

- Enfermaria Veterinária: constituída por cinco unidades, sendo elas a Enfermaria veterinária para grandes animais (equinos, asininos e bovinos), Enfermaria veterinária para animais de fazenda (aves e suínos), Enfermaria veterinária para caninos, Enfermaria veterinária para felinos e Enfermaria veterinária para animais selvagens; com equipamentos adequados a essa função, devendo estar cada unidade sob a supervisão de um médico veterinário especialista. Essas unidades devem garantir a estabilização, monitorização e recuperação total dos animais vitimados.

- $\quad$ Armazém de insumos veterinários: constituído por uma unidade e equipamentos logísticos de apoio, devendo estar sob a supervisão de um médico veterinário com formação e/ou experiência em gestão/logística hospitalar. A função desse setor consiste em identificar e classificar os produtos hospitalares e todos os demais que serão utilizados na identificação dos animais, alimentação, vacinação, desinfecção dentre outros.

- $\quad$ Unidade Médico-Cirúrgica Veterinária (UMCV): constituída por uma unidade destinada ao preparo cirúrgico, uma unidade pré-operatória, um bloco cirúrgico e uma unidade pós-operatória. Devendo estar sob a supervisão de um médico veterinário cirurgião geral, um ortopedista, um anestesista e um intensivista. A função desse setor consiste na realização de cirurgias de média a alta complexidade.

- $\quad$ Unidade Diagnóstica e Laboratorial: constituída por uma unidade destinada à coleta e envio de amostras para avaliações laboratoriais e de exames de imagem, devendo estar sob a supervisão de um médico veterinário patologista clínico e um em diagnóstico por imagem.

- $\quad$ Unidade de Liberação e Adoção de Animais Resgatados: constituída por uma unidade destinada a liberar os animais vitimados ao(s) seu(s) respectivo(s) tutor(es) ou para adoção, devendo estar sob a supervisão de um médico veterinário com experiência na saúde do coletivo.

\section{Ciclo de gestão da emergência}

A gestão da emergência é um processo contínuo desenvolvido para prevenir, mitigar e preparar a resposta e recuperação de um incidente que ameaça a vida, a propriedade, operações ou ambiente (National Fire Protection Association, 2013). A Figura 1 demonstra o tipo de gestão adotada durante o incidente ocasionado pela mineradora Samarco em Mariana-MG, Brasil, e que serve como balizamento para o processo de intervenções futuras. Infelizmente no caso em questão não foram adotadas medidas pré-desastre ou catástrofe. Vale salientar que houve aprendizado em todas as etapas que nos permite traçar planos de treinamentos e ações para intervenções futuras.

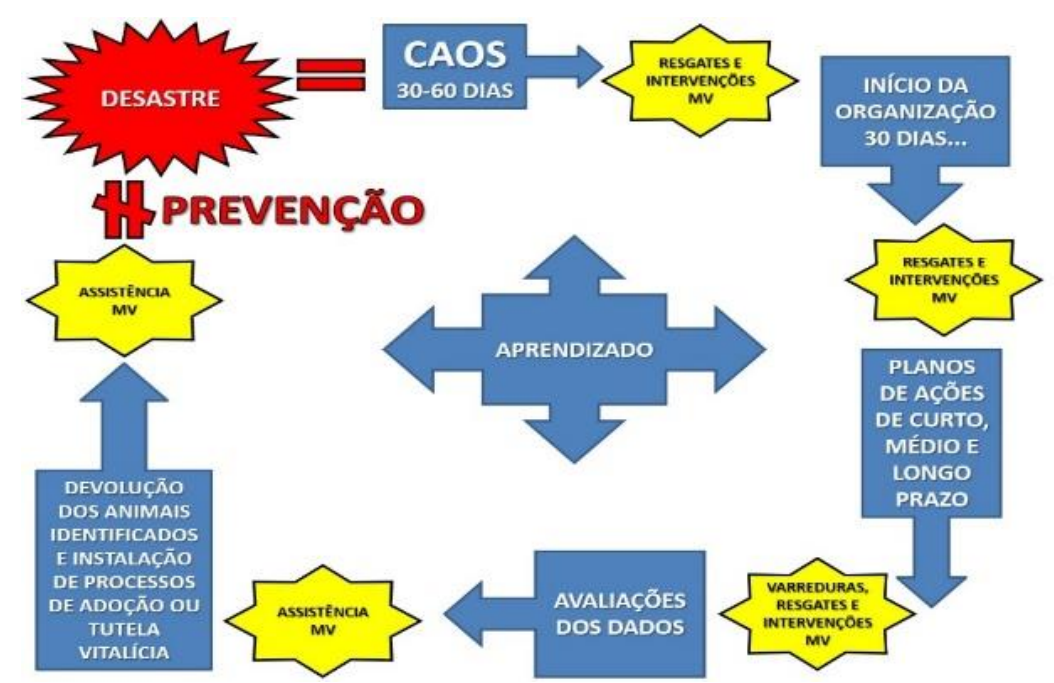

Figura 1. Ciclo de gestão de emergência Médica Veterinária (MV) adotado durante a Megacatástrofe após o rompimento da Barragem de Fundão - Samarco (Mariana, MG, Brasil) 
A natureza cíclica determina a necessidade de ações anteriores e posteriores ao agravo. O ciclo pode ser dividido em três fases: pré-desastre ou catástrofe, durante o desastre ou catástrofe e pós-desastre ou catástrofe. O pré-desastre consiste nas medidas efetivas a serem adotadas de modo a prevenir e preparar a população para o possível incidente. Durante o desastre serão adotadas medidas para mitigar os efeitos ocasionados pelo desastre ou catástrofe. Nesse período se dá ênfase a magnitude, espécies e número de animais afetados, taxa de mortalidade e morbidade, tipos de lesões e doenças, danos às infraestruturas que alojavam os animais, qualidade e fornecimento de comida e água. Já o pós-desastre é dividido em recuperação, reabilitação e devolução/adoção dos animais. Outro ponto de extrema relevância é o apoio médico veterinário aos cães de busca e salvamento utilizados pelas forças especiais, dividindo uma responsabilidade cívica de auxiliar estes heróis quadrúpedes, tão indispensáveis e que tanto faz em prol das vítimas de desastres.

\section{Conclusão}

Visto que a emergência exige rapidez de resposta, esse não é o melhor momento para se iniciar um processo de aprendizagem entre os profissionais que irão intervir. Dentro dessa ótica necessitamos desenvolver um programa de aperfeiçoamento veterinário para a atuação em desastres como a criação da Sociedade Brasileira de Medicina Veterinária para Casos de Desastres que seria formada por médicos veterinários e outros profissionais responsáveis pela intervenção em desastres que envolvam animais, ou o homem, no caso das zoonoses.

É importante criar Centros Veterinários para a Prevenção em Casos de Desastres para atuarem como referência em todos os estados da federação sob a égide do Conselho Federal de Medicina Veterinária, Ministério da Saúde e do Ministério da Agricultura, Pecuária e Abastecimento. Considera-se que um profissional ligado às emergências deva ser versátil e extremamente apto às intervenções propostas. É fundamental para o veterinário que vai atuar nessas situações, a experiência clínica profissional, e para as intervenções, a utilização de diferentes dispositivos clínicos, tanto individuais como em equipe. E acrescentaria a importância da experiência profissional em saúde pública como essencial para estas práticas, já que proporciona o trabalho com grandes populações e uma visão integral de saúde do coletivo.

A inclusão no Código de Ética Profissional do Médico Veterinário de um artigo e que esse defina como um dos seus deveres fundamentais, a prestação de serviços profissionais em situações de calamidade pública ou de emergência, sem visar benefício pessoal. Ou seja, esse serviço poderá ser prestado de forma gratuita, inclusive mediante trabalho voluntário. Tal inclusão se torna necessária pela responsabilidade social que a Medicina Veterinária presta à sociedade, e deve ser encarado com seriedade e profissionalismo, seguindo todas as demais responsabilidades previstas pelo Código de Ética do Médico Veterinário.

São vários os relatos ao longo da história que salientam a importância dos serviços Médicos Veterinários em situação de catástrofe e é, em grande medida, a dependência interespecífica existente entre homem e as demais espécies animais. Tal dependência reforça o conceito "One Health" tornando a dissociação entre a saúde humana e a saúde animal um pensamento para uma sociedade dependente do ecossistema em que se insere.

Os tutores têm um papel central na defesa dos seus animais em situação de catástrofe, portanto, é de suma importância que seja elaborado um manual com um plano de emergência direcionado para as famílias com animais de companhia, trabalho e produção de forma a dar um balizamento às suas necessidades, caso ocorra algum incidente.

\section{Referências bibliográficas}

Aires, C. A. M., Araujo, C. F. M., Nobre, M. L., Rusak, L. A., Assis, U. G., Lopéz, D. C. M. \& Portilho, M. M. (2015). Biossegurança em transporte de material biológico no âmbito nacional: um guia breve. Revista Pan-Amazônica de Saúde, 6(2):1-9.

Bandeira, R. (2008). Medicina de Catástrofe. Porto, Portugal: Editora da Universidade do Porto.

Guha-Sapir, D., Hoyos, P. \& Below, R. (2013). Annual disaster statistical review. Acesso em: 06 março de 2016, disponível em: http://cred.be/sites/default/files/ADSR_2013.pdf. 
Irvine, L. (2009). Conclusion: Noah's Task. In L. Irvine (Ed.), Filling the Ark: Animal welfare in disasters. Philadelphia, usa: Temple University Press.

National Fire Protection Association. (2013). Standard on Disaster/Emergency Management and Business Continuity Programs. Acesso em 15 de julho de 2018, disponível em: https://www.nfpa.org/assets/files/AboutTheCodes/1600/1600-13-PDF.pdf.

PAHO - Emergency Health management after Natural Disaster (1981).

Resolução $\mathrm{n}^{\circ} 1000$ do CFMV de 11 de maio de (2012). $<$ http://portal.cfmv.gov.br/legislacao/index?titulo-descricaoresumo=Resolu\%C3\% A7\% $3 \% \mathrm{~A} 30+n \% \mathrm{C} 2 \% \mathrm{BA}+1000+\mathrm{CFMV}+\mathrm{de}+11+\mathrm{de}+$ maio+de+2012\&cate gorias $\% 5 \mathrm{~B} \% 5 \mathrm{D}=$ todas\&ordenacao=numero $>$ Acesso em 2 de setembro de 2019.

Ryan, J. M. (2008). Triage: principles and pressures. European Journal of Trauma and Emergency Surgery, 34(5):1-6.

Sundnes, K. O. \& Birnbaum, M. L. (2003). Health disaster management: Guidelines for evaluation and research in the Utstein style. Prehospital and Disaster Medicine, 17(3):1-14.

United States Departament of Health \& Human Services. (2015). National Veterinary Response Team. Acesso em: 06 março de 2016, disponível em: http://www.phe.gov/preparedness/responders/ndms/teams/pages/nvrt.aspx.

Ventura, R. (2011). Os impactos das emergências e dos desastres na política de Assistência Social. Conselho Federal de Psicologia, 10050-54.

Vieira, J. F. M. (2016). Medicina veterinária de desastres e catástrofes. Master of Science, Universidade de Lisboa, Lisboa, Portugal.

Wingfield, E. W. (2009). Veterinary triage. In W. E. Wingfield \& S. B. Palmer (Eds.), Veterinary disaster response (pp. 111-121). Iowa, USA: Wiley-Blacwell.

Wingfield, E. W. \& Palmer, B. S. (2009). Veterinary triage. In W. E. Wingfield \& S. B. Palmer (Eds.), Veterinary disaster response (pp. 111-121). Iowa, USA: Wiley-Blacwell.

Recebido: 2 de setembro, 2019.

Aprovado: 1 de outubro, 2019.

Publicado: 26 de novembro, 2019.

Licenciamento: Este artigo é publicado na modalidade Acesso Aberto sob a licença Creative Commons Atribuição 4.0 (CC-BY 4.0), a qual permite uso irrestrito, distribuição, reprodução em qualquer meio, desde que o autor e a fonte sejam devidamente creditados. 